\title{
Changing disease patterns in patients with AIDS in a referral centre in the United Kingdom: the changing face of AIDS
}

\author{
B S Peters, E J Beck, D G Coleman, M J H Wadsworth, O McGuinness, J R W Harris, \\ A J Pinching
}

\begin{abstract}
Objective-To study the changes in morbidity, mortality, and survival patterns in a population of patients with AIDS in the United Kingdom from 1982 to 1989.

Design-A retrospective analysis of inpatient and outpatient records of patients with AIDS.

Subjects - 347 Patients with AIDS, predominantly homosexual or bisexual men.

Setting-Departments of immunology and genitourinary medicine, St Mary's Hospital, London.
\end{abstract}

Main outcome measures-Presenting diagnosis of AIDS, occurrence of other opportunist diseases, cause of death, and survival since AIDS was diagnosed, in particular for those patients with Pneumocystis carinii pneumonia or Kaposi's sarcoma.

Results - The overall proportion of patients who developed $P$ carinii pneumonia dropped from $56 \%$ $(20 / 36)$ in 1984 to $24 \%(46 / 194)$ in 1989 , although it has remained the index diagnosis in about half of new patients. Kaposi's sarcoma has decreased as index diagnosis from $30 \%(20 / 67)$ to $20 \%(15 / 74)$ over the same period, though the prevalance has remained constant at around $35 \%$. P carinii pneumonia accounted for $46 \%(16 / 35)$ of known causes of death in 1986 but only 3\% (1/31) in 1989. Conversely, deaths due to Kaposi's sarcoma rose from 14\% (1/7) to 32\% (10/31) between 1984 and 1989.

Department of

Immunology, St Mary's

Hospital Medical School, London W2 1PG

B S Peters, MRCP, research and clinical registrar

D G Coleman, data manager

O McGuinness, BSC, medical student

A J Pinching, FRCP, reader

Academic Department of Public Health, St Mary's Hospital Medical School, London W2 1PG

E J Beck, MFPHM, lecturer

M J H Wadsworth, MSC, senior lecturer

\section{Department of}

Genitourinary Medicine, St

Mary's Hospital Medical

School, London W2 1PG

J R W Harris, FRCP,

consultant physician

Correspondence to: $\mathrm{Dr}$ Peters.

BMf 1991;302:203-7
Lymphoma accounted for an increased proportion of deaths among these patients with $16 \%(5 / 31)$ of deaths in 1989. Their median survival increased from 10 months in 1984-6 to 20 months in 1987.

Conclusions - The changing patterns of disease in patients with AIDS have important implications both for health care provision and future medical research. Medical and nursing provision must be made for the increased morbidity of these diseases and the increased survival of these patients. Research should now be directed towards developing effective treatments for the opportunist infections which are currently more difficult to treat, the secondary malignancies of AIDS, as well as more effective immunorestorative treatments. Future changes in disease patterns must be recognised at an early stage so that resources can be adequately planned and allocated.

\section{Introduction}

In its first decade the clinical profile of AIDS has been extensively reported and its main complications are now well recognised. The incidence of specific diseases varies according to locality owing to the varying prevalence of potential opportunist pathogens. Broadly similar patterns are seen in temperate regions, however. A diminishing incidence of Kaposi's sarcoma among homosexual men in the United States ${ }^{1-3}$ and the emergence of new opportunist pathogens, such as leishmania, ${ }^{4}$ have now been reported, and reduced mortality from pneumocystis pneumonia has also recently been found in the United States.

As experience has grown the management of some of the main opportunist diseases may have been improved by earlier diagnosis, better treatment, and increased use of prophylaxis. ${ }^{6-8}$ Such interventions are increasingly likely to affect the clinical profile of AIDS in terms of morbidity and mortality. Improved diagnosis and treatment of some diseases, however, may also highlight others. In particular, diseases that are more amenable to current treatments may be gradually replaced by those that are more resistant to treatment. The widespread introduction of zidovudine in 1987, an antiretroviral drug which slows the rate of progression of the underlying disease, is prolonging survival. ${ }^{8-1}$ The resulting longer duration of immunodeficiency may also influence the frequency and progression of other secondary infections or tumours.

To study the improving prognosis for patients with AIDS and to identify changing morbidity and mortality trends over time we conducted a retrospective analysis of patients managed at our centre from 1982 to 1989. This type of analysis helps to ensure that new approaches to care and treatment are adapted to the changing profile of the disease.

\section{Methods}

Patients - We studied the patients with AIDS who attended our hospital regularly (usually monthly or more often) for their clinical management between October 1982 and 31 December 1989. We excluded 14 patients with incomplete or unavailable notes, five who were lost to follow up, and 47 who lived elsewhere but sought advice for problems that arose while temporarily in our locality. This left 347 patients for study (342 men and five women); 285 were from the London metropolitan area; 48 were from elsewhere in the United Kingdom; and 14 were foreign nationals, half of them from sub-Saharan Africa. The racial composition was 330 white, 10 Afro-Caribbean, four Hispanic, and three Asian. Most (310) were homosexual men, 23 were bisexual men, and 14 were heterosexual men; seven were intravenous drug users. The average age at diagnosis was 37.5 years, which remained constant over time (table I). Since April 1987 $79 \%$ of patients with AIDS at our hospital have elected to be treated with zidovudine. The revised Centres for Disease Control (CDC) classification was used for defining AIDS. ${ }^{12}$

Trial design is shown in figure 1 .

Data collected-Information recorded included sex, sexual orientation, intravenous drug use, date of birth, date that AIDS was diagnosed, presenting illness for 
AIDS, date that Kaposi's sarcoma was diagnosed, and each episode of pneumocystis pneumonia. The use of zidovudine and prophylaxis for pneumocystis pneumonia was noted. The date and cause of death were noted using clinical information with pathological confirmation. Information on necropsy, including needle necropsy, was obtained if performed. If all or most of these criteria were met then known cause of death was recorded. When doubt persisted then cause of death was recorded as unknown, and the organ system failures resulting in death were recorded instead.

Statistical analysis - The collected data were analysed using several methods including Spearman's rank correlation and the log rank test ${ }^{13}$ for the survival

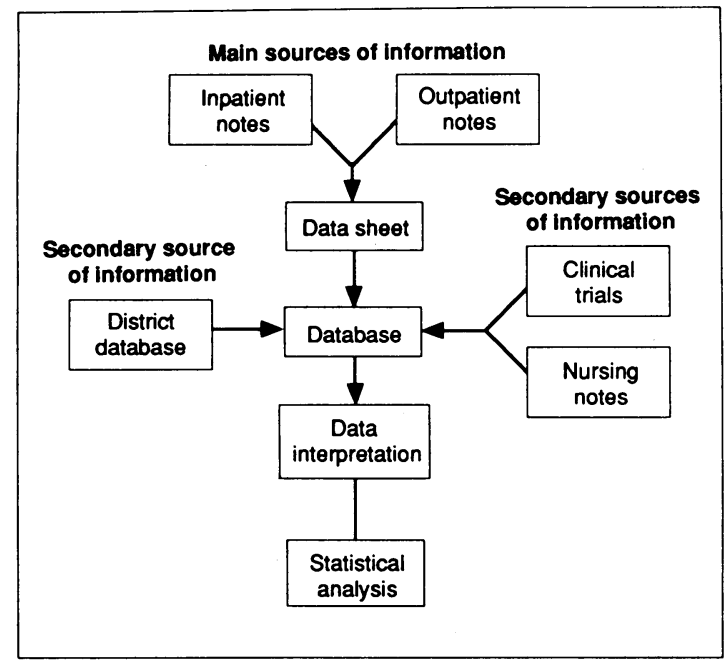

FIG 1-Design of study

TABLE I-Details of patients with AIDS by year of diagnosis. Figures are number of patients with numbers of intravenous drug users in brackets

\begin{tabular}{|c|c|c|c|c|c|c|c|c|}
\hline \multirow[b]{2}{*}{ Patients } & \multicolumn{8}{|c|}{ Year of diagnosis } \\
\hline & $1982-3$ & 1984 & 1985 & 1986 & 1987 & 1988 & 1989 & Total \\
\hline $\begin{array}{l}\text { Men } \\
\text { Women }\end{array}$ & 9 & $\begin{array}{r}29 \\
1\end{array}$ & 23 & 67 & 79 & 65 & $\begin{array}{r}70 \\
4 \\
\end{array}$ & $\begin{array}{r}342 \\
5 \\
\end{array}$ \\
\hline Total & 9 & 30 & 23 & 67 & 79 & 65 & 74 & 347 \\
\hline $\begin{array}{l}\text { Homosexual } \\
\text { Heterosexual } \\
\text { Bisexual } \\
\text { Mean age (years) } \\
\text { Age range (years) }\end{array}$ & $\begin{array}{c}35 \cdot 1 \\
20-52\end{array}$ & $\begin{array}{c}25 \\
1 \\
4 \\
40 \cdot 7 \\
24-59\end{array}$ & $\begin{array}{c}22 \\
1 \\
38 \cdot 1 \\
22-7 i\end{array}$ & $\begin{array}{c}57(1) \\
2(1) \\
8 \\
38 \cdot 5 \\
20-60\end{array}$ & $\begin{array}{c}71(1) \\
3(1) \\
5(1) \\
38 \cdot 5 \\
22-62\end{array}$ & $\begin{array}{c}57 \\
4(2) \\
4 \\
36 \cdot 8 \\
24-57\end{array}$ & $\begin{array}{c}69 \\
4 \\
1 \\
38 \cdot 1 \\
26-57\end{array}$ & $\begin{array}{c}310(2) \\
14(4) \\
23(1) \\
37 \cdot 5 \\
20-71\end{array}$ \\
\hline
\end{tabular}

analyses, using Statistical Analysis System (SAS) software.

Treatment protocols-The overall approach to treatment and diagnosis throughout the study has been consistent and conducted according to prospectively defined protocols. There have been few major changes in treatment strategy. For example, pneumocystis pneumonia has been treated with high dose cotrimoxazole as first line treatment and dapsone and trimethoprim if intolerance occurred; parenteral pentamidine has rarely been used. Since 1988 a short course of high dose corticosteroids has been used to treat patients with pneumocystis pneumonia who deteriorate despite antimicrobial treatment. ${ }^{14}$ Secondary prophylaxis was not used initially but since 1988 has been offered to most patients in the form of fortnightly nebulised pentamidine, weekly Fansidar, or weekly dapsone and pyrimethamine. Primary prophylaxis has not been used routinely. For Kaposi's sarcoma low dose radiotherapy has been used for visible lesions and chemotherapy has been restricted to those with visceral disease or rapidly progressing cutaneous or lymphatic disease. Initially etoposide was our first line treatment (1985-6), but the treatment of choice sine 1987 has been vincristine and bleomycin. From April 1987 patients with AIDS were offered zidovudine with the initial exception of patients with limited Kaposi's sarcoma and no other HIV related features. Although most eligible patients took zidovudine, a few elected not to do so, providing a concurrent but uncontrolled comparative group. Some of these entered studies on the drug AL721 and have been reported on elsewhere. ${ }^{15}$

\section{Results}

\section{MORBIDITY}

The most common index diagnosis for AIDS was pneumocystis pneumonia (176) followed by Kaposi's sarcoma (86) (table II). The ratio of patients presenting with pneumocystis pneumonia compared with those presenting with Kaposi's sarcoma increased over time $(\mathrm{R}=0.71, \mathrm{p}=0.05)$. Patients with AIDS presenting with pneumocystis pneumonia stabilised at around $57 \%(38 / 67)$ in 1986 and those presenting with Kaposi's sarcoma decreased from $30 \%(20 / 67)$ in 1986 to $20 \%$ (15/74) in 1989 (table II). Similarly, the proportion of patients with AIDS alive each year who developed Kaposi's sarcoma declined from $24 \%(8 / 33)$ in 1984 to $16 \%(25 / 156)$ in 1989 . The prevalence of Kaposi's sarcoma in patients with AIDS alive during the year

TABLE II - Indices of morbidity and mortality of patients with AIDS for each year of study

\begin{tabular}{|c|c|c|c|c|c|c|c|c|}
\hline \multirow[b]{2}{*}{ Patient } & \multicolumn{8}{|c|}{ Year of diagnosis } \\
\hline & $1982-3$ & 1984 & 1985 & 1986 & 1987 & 1988 & 1989 & Total \\
\hline New patients & 9 & 30 & 23 & 67 & 79 & 65 & 74 & 347 \\
\hline Deaths & 3 & 10 & 22 & 39 & 43 & 39 & 41 & 197 \\
\hline \multicolumn{9}{|l|}{ No (\%) with index diagnosis: } \\
\hline Pneumocystis carinii pneumonia & $2 / 9(22)$ & $12(40)$ & $8(35)$ & $38(57)$ & $41(52)$ & $37(56)$ & $38(52)$ & $176(51)$ \\
\hline Kaposi's sarcoma & $3(33)$ & $5(17)$ & $7(30)$ & $20(30)$ & $22(28)$ & $14(22)$ & $15(20)$ & $86(25)$ \\
\hline Others ${ }^{\star}$ & $4(45)$ & $13(43)$ & $8(35)$ & $9(13)$ & $16(20)$ & $14(22)$ & $21(28)$ & $85(24)$ \\
\hline Live patients/year & & 36 & 49 & 94 & 134 & 156 & 194 & \\
\hline $\begin{array}{c}\text { No (\%) of patients with } \geqslant 1 \text { episode } \\
\text { Pneumocystis carinii pneumonia }\end{array}$ & & $20 / 36(56)$ & $14 / 49(29)$ & $48 / 94(51)$ & $57 / 134(43)$ & $43 / 156(28)$ & $46 / 194(24)$ & \\
\hline No of new patients developing Kaposi's & & & & & & & & \\
\hline $\begin{array}{l}\text { sarcoma } \\
\text { No of live patients with Kaposi's }\end{array}$ & & 8 & 9 & 22 & 32 & 24 & 25 & \\
\hline sarcoma & & 11 & 18 & 31 & 51 & 55 & 63 & \\
\hline Prevalence of Kaposi's sarcoma & & $31 \%(11 / 36)$ & $37 \%(18 / 49)$ & $33 \%(31 / 94)$ & $38 \%(51 / 134)$ & $35 \%(55 / 156)$ & $32 \%(63 / 194)$ & \\
\hline Incidence of Kaposi's sarcoma $†$ & & $24 \%(8 / 33)$ & $23 \%(9 / 40)$ & $26 \%(22 / 85)$ & $28 \%(32 / 115)$ & $19 \%(24 / 125)$ & $16 \%(25 / 156)$ & \\
\hline \multicolumn{9}{|l|}{ No $(\%)$ of patients with known cause of death: } \\
\hline Pneumocystis carinii pneumonia & $1 / 3(33)$ & $2 / 7(29)$ & $6 / 7(35)$ & $16 / 34(46)$ & $12 / 30(40)$ & $7 / 26(27)$ & $1 / 31(3)$ & $45 / 149(30)$ \\
\hline Kaposi's sarcoma & & $1 / 7(14)$ & $2 / 17(12)$ & $4 / 34(11)$ & $7 / 30(23)$ & $7 / 26(27)$ & $10 / 31(32)$ & $31 / 149(21)$ \\
\hline Lymphoma & & & $1 / 17(6)$ & & $3 / 30(10)$ & $3 / 26(12)$ & $5 / 31(16)$ & $12 / 149(8)$ \\
\hline Other ${ }^{\star}$ & $2 / 3(67)$ & $4 / 7(57)$ & $8 / 17(47)$ & $15 / 34(43)$ & $8 / 30(27)$ & $9 / 26(34)$ & $15 / 31(49)$ & $61(41)$ \\
\hline Unknown & & 3 & 5 & 4 & 13 & 13 & 10 & 48 \\
\hline
\end{tabular}

^ Includes cryptosporidium, cryptococcal, toxoplasma, cytomegalovirus infection, oesophageal candidiasis, progressive multifocal leucoencephalopathy, Mycobacterium avium intracellulare or extrapulmonary tuberculosis infection, and HIV encephalopathy.

The proportion of live patients without Kaposi's sarcoma during the year who developed Kaposi's sarcoma. 

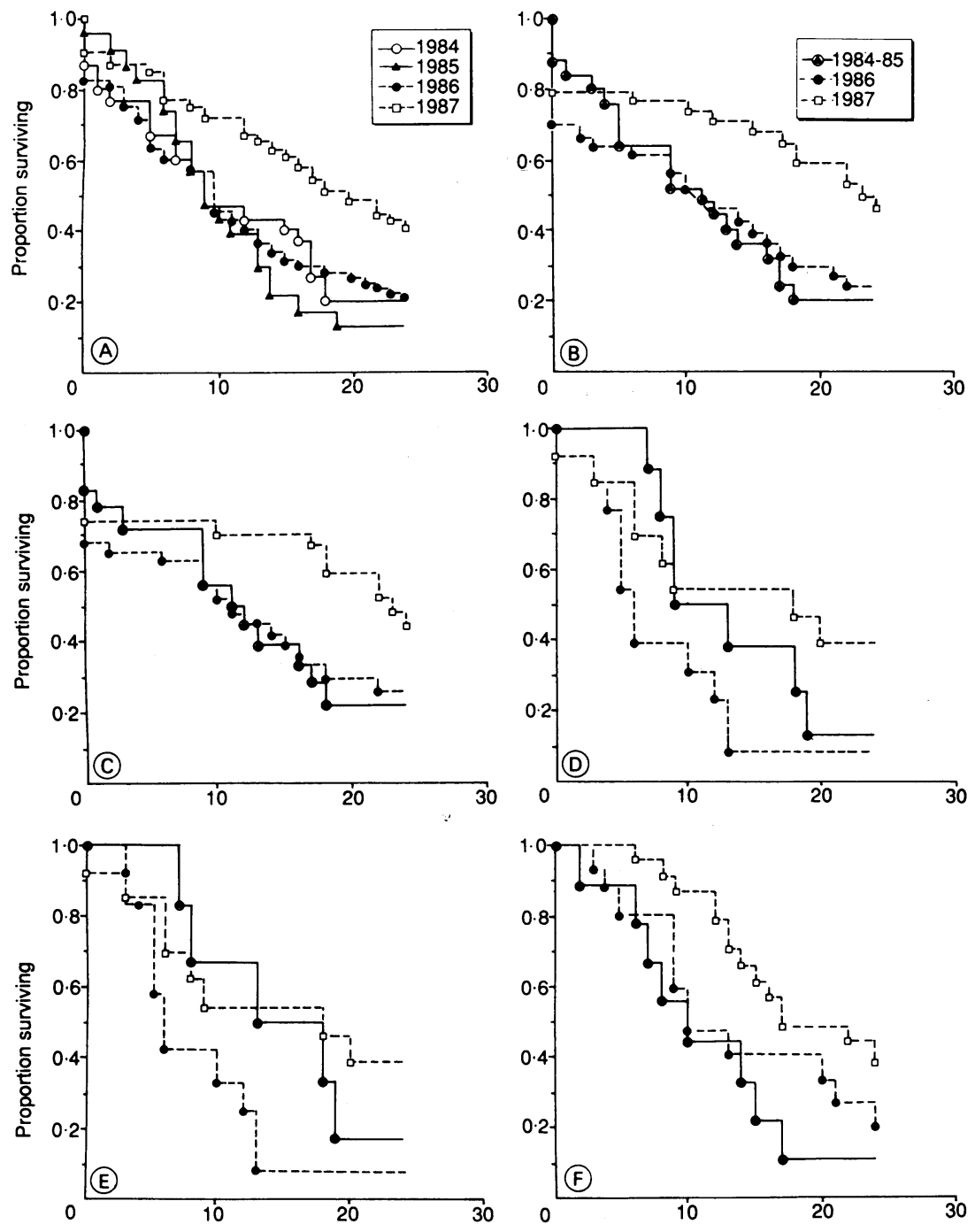

Median survival time (months)

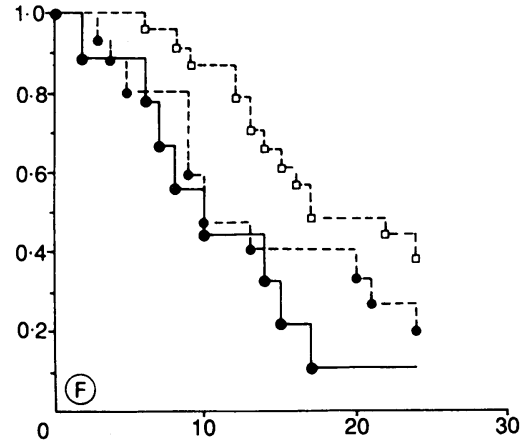

Median survival time (months)

FIG 2-Two year survival curves for patients with AIDS by year of diagnosis between 1984 and 1987 for: (a) total population; (b) patients with Pneumocystis carinii pneumonia; (c) patients with $P$ carinii pneumonia as index diagnosis; (d) patients with Kaposi's sarcoma; (e) patients with Kaposi's sarcoma as index diagnosis; $(f)$ patients with P carinii pneumonia and Kaposi's sarcoma

remained constant around $35 \%$ between 1984 and 1989 (table II). The proportion of patients with AIDS with one or more episodes of pneumocystis pneumonia diagnosed decreased from $56 \%(20 / 36)$ in 1984 to $24 \%$ (46/194) in 1989.

Complications of disseminated Kaposi's sarcoma have been increasingly seen as causes of emergency admission to our wards and include: pulmonary Kaposi's sarcoma with secondary bacterial pneumonias, gastrointestinal Kaposi's sarcoma with obstruction or haemorrhage, and lymphatic infiltration with oedema, skin ulceration, and infection. In addition, chemotherapy for Kaposi's sarcoma was one of the commonest causes of planned hospital admissions, secondary only to transfusions for zidovudine related anaemia.

Other AIDS related conditions were diagnosed in our patients as follows: cytomegalovirus retinal or bowel disease in $25 \%$ ( 87 patients), oesophageal candidiasis in $13 \%$ (45), cerebral toxoplasmosis in $10 \%$ (35), Mycobacterium avium complex infections in 10\% (35), cryptosporidiosis in $9 \%$ (31), extrapulmonary tuberculosis in $7 \%$ (24), cryptococcal meningitis in $4 \%$ (14), lymphoma in $5 \%(17)$, and progressive multifocal leucoencephalopathy in $2 \%(7)$.

\section{MORTALITY}

From 1982 to 1989,197 patients died, $57 \%$ of the total population. Of the 149 with a known cause of death the highest number (45) died of pneumocystis pneumonia, and 31 of Kaposi's sarcoma (table II). Lymphoma and toxoplasmosis each accounted for 12 known deaths. The ratio of patients with AIDS who died from pneumocystis pneumonia compared with those who died from Kaposi's sarcoma decreased over time $(\mathrm{r}=-0.77, \mathrm{p}<0.05)$. Pneumocystis pneumonia constituted $46 \%(16 / 35)$ of known causes of death in 1986 and dropped to $3 \%(1 / 31)$ in 1989 (table II). Conversely, though $14 \%$ (1/7) of patients with AIDS in 1984 died of Kaposi's sarcoma this increased to $32 \%$ $(10 / 31)$ in 1989. During the past three years there has also been a progressive increase in the number of patients dying from lymphoma, predominantly cerebral lymphoma. In 1989 this caused $16 \%(5 / 31)$ of deaths of known cause (table II). The number of deaths as a proportion of all patients alive also decreased from 1984 to 1989.

In 48 patients death could not be accurately attributed to a specific cause. Most of these patients had longstanding AIDS and died from multisystem organ failure. Only six patients, all of whom were too ill to have bronchoscopy, died of respiratory failure, possibly due to $P$ carinii pneumonia. None of these deaths, however, occurred in 1989. Ten patients had predominantly central nervous system signs or symptoms leading up to their death with evidence of cerebral space occupying lesions in six cases. No evidence of cryptococcal or toxoplasma infections was found on lumbar puncture and none responded to treatment. Progressive multifocal leucoencephalopathy or cerebral lymphoma were the most likely causes of death, although cytomegalovirus or herpes simplex encephalitis could not be ruled out. Hepatic failure was pronounced in six patients, but this usually occurred before death.

\section{SURVIVAL ANALYSIS}

Two year survival curves were calculated for the 199 new patients diagnosed from 1984 to 1987 . The median survival times were similar for the 1984-6 cohorts (9-10 months) but were significantly longer for the 1987 cohort (20 months; fig 2a, log rank test $\chi^{2}=14 \cdot 8$, $\mathrm{p}<0.002)$. Survival analyses were subsequently performed on the 92 patients who developed pneumocystis pneumonia at any time during their course as a patient as well as those who presented with pneumocystis pneumonia (figs $2 b$ and $2 c$ ). Significant differences were again found between the patients with pneumocystis pneumonia diagnosed before 1987 (median survival time 11 months) and the 1987 cohort (median survival time 23 months; log rank test $\left.\chi^{2}=7 \cdot 4, p<0.05\right)$. The 76 patients with pneumocystis pneumonia as index diagnosis had similar survival curves to the total pneumocystis pneumonia group but because of reduced numbers these were not statistically significant (log rank test $\chi^{2}=4 \cdot 5, p=0 \cdot 11$ ). Survival curves were also calculated for patients with Kaposi's sarcoma either at any time during their disease course or as the index diagnosis. Median survival times for patients with Kaposi's sarcoma in the 1984-6 cohorts were 6-12 months and 18 months for the 1987 cohort (fig 2d) which was of borderline significance (log rank test $\left.\chi^{2}=5 \cdot 5, p=0.06\right)$. Thirty four patients were included in this category and this was further reduced to 31 in the group with Kaposi's sarcoma as index diagnosis (fig $2 \mathrm{e}$; $\log$ rank test $\chi^{2}=4 \cdot 8, \mathrm{p}=0 \cdot 09$ ). Finally, survival rates were calculated for the 47 patients who developed both pneumocystis pneumonia and Kaposi's sarcoma during their illness course. Again, the survival rate of those groups diagnosed before 1987 (median survival time 10 months) was shorter than those in the 1987 cohort (median survival time 17 months) and the difference was of borderline significance $\left(\log\right.$ rank test $\chi^{2}=5 \cdot 7, p<0 \cdot 06$; fig $\left.2 f\right)$. The 
pattern of survival of the patients with Kaposi's sarcoma was similar to that of those patients with pneumocystis pneumonia, but it was more difficult to draw firm conclusions because of the smaller numbers.

Of 63 patients who had AIDS diagnosed between April and December 1987, 47 were started on zidovudine. Five died within one month before zidovudine could be offered to them, and 11 elected not to take it. All but one of the non-zidovudine group also elected not to take prophylaxis for pneumocystis pneumonia. The median survival time of those taking zidovudine was greater than 24 months compared with a median survival for the non-zidovudine group of seven months (log rank test $\left.\chi^{2}=13 \cdot 2, p=0 \cdot 0003\right)$

\section{Discussion}

The clinical profile and prognosis of our patients with AIDS are changing. The incidence of Kaposi's sarcoma in our United Kingdom population has started to show a decline similar to that previously found in patients in the United States,,$^{1-3}$ while pneumocystis pneumonia has remained the index diagnosis for AIDS in roughly half. The median survival time for our patients with AIDS has doubled since 1987, from 10 to 20 months, having remained stable in previous years. The improved survival applies to all main diagnostic groups. Mortality due to pneumocystis pneumonia has shown a striking recent fall, and an increasing proportion of deaths are now due to Kaposi's sarcoma and cerebral lymphoma.

The considerable reduction in mortality due to pneumocystis pneumonia largely accounts for the improved outlook among our patients. Several factors are likely to have influenced this reduction, including zidovudine treatment. A reduction in mortality due to pneumocystis pneumonia was found in a North American study before the introduction of zidovudine, ${ }^{16}$ suggesting that other factors, such as earlier presentation or earlier diagnosis and treatment, ${ }^{17}$ have been important. The finding that pneumocystis pneumonia mortality primarily relates to the extent of pneumonitis at presentation supports this. ${ }^{18}$ Early studies on zidovudine suggested that it not only reduced the frequency of opportunist infections in the first six months of use but also reduced their severity. ${ }^{9}$ It is unclear to what extent the increased use of pneumocystis pneumonia prophylaxis and the introduction of corticosteroids for severe pneumocystis pneumonia have contributed to the falling mortality in our series. Prospective studies are required to elucidate fully the role of these treatments.

Although the numbers of specific opportunist infections other than $P$ carinii are often too small for separate analysis, some trends can also be seen. As the morbidity of some conditions decreases and survival times improve then those disorders refractory to treatment become more manifest. These include cryptosporidiosis and progressive multifocal leucoencephalopathy, for which no effective antimicrobial agents are available, and Mycobacterium avium intracellulare infections and late relapses of cryptococcal meningitis, where current treatment has limited efficacy.

Changes have also been seen in both the presentation and prognosis of our patients with Kaposi's sarcoma. The improved survival we have seen with Kaposi's sarcoma may partly be due to a fall in deaths due to opportunist infections. A consequence of this longer survival, however, is that more patients are now developing extensive visceral Kaposi's sarcoma and are dying because of systemic disease: Kaposi's sarcoma was the commonest cause of death in 1989. Lymphoma, especially cerebral lymphoma, has also recently emerged as a more common cause of death among our patients. This may again be related to increased survival: the importance of the duration of immunosuppression in determining the incidence of lymphomas has already been reported in patients with renal transplants. ${ }^{19}$ It is important to note that other malignancies may appear as a consequence of more prolonged immunosuppression. ${ }^{20}$

A contributory factor in improving survival in our patients has been the introduction of zidovudine in 1987. A placebo controlled study showed a considerable reduction in early mortality' and subsequent studies have confirmed these findings. ${ }^{1011}$ Improvement in survival in our study occurred in the year that zidovudine was widely introduced. Possibly the use of zidovudine contributed to this, especially considering the poor median survival among our patients who elected not to use it, although they also tended not to use other treatments, such as pneumocystis pneumonia prophylaxis.

Considerable improvements in the outlook for patients with AIDS can be expected in the future but to help achieve this we need to change our priorities as the profile of the disease changes. Strategies for treating opportunist disease will need increasingly to focus on those diseases now causing highest morbidity and mortality. More effective antiretroviral drugs may be developed in the future but at present optimising the timing and dose of zidovudine remains an important goal if we are to maximise its benefits and minimise its toxicity. ${ }^{21}$

The improved survival of patients with AIDS and the improved quality of life that has accompanied it are encouraging. The increased requirements for care and the changing patterns of disease, however, will need to be incorporated into the forward planning of resources. For example, the increased morbidity due to opportunist tumours has not only affected our hospital practice but has also placed increased demands on community and hospice services. It will be essential to continue to monitor the changing clinical profile of AIDS at local and regional levels to target future clinical and research priorities.

We thank Dr Harold Jaffe for encouragement and constructive comments. DGC was supported by the North West Thames Regional Health Authority HIV Service Research Fund.

1 Selik RM, Starcher ET, Curran JW. Opportunistic diseases reported in AIDS patients: frequencies, associations and trends. AIDS 1987;1:175-82.

2 Rutherford GW, Schwarcz SK, Lemp GF, et al. The epidemiology of AIDSrelated Kaposi sarcoma in San Francisco. I Infect Dis 1989;159:567-71.

3 Beral V, Peterman TA, Berkelman RL, Jaffe HW. Kaposi's sarcoma among persons with AIDS: a sexually transmitted infection? Lancet 1990;335: 123-8.

4 Peters BS, Fish D, Golden R, Evans DA, Bryceson ADM, Pinching AJ. Visceral leishmaniasis in HIV infection and AIDS: clinical features and response to therapy. $Q \mathcal{F} \mathrm{Med}$ (in press).

5 Harris JE. Improved short-term survival of AIDS patients initially diagnosed with Pneumocystis carinii pneumonia 1984 through 1989. JAMA 1990;263: 397-401.

6 Thomas S, O'Doherty M, Bateman N. Pneumocystis carinii pneumonia. $B M \mathcal{F}$ 1990;300:211-2.

7 Fischl MA, Dickinson GM. Safety and efficacy of sulfamethoxazole and trimethoprim chemoprophylaxis for Pneumocystis carinii pneumonia in AIDS. FAMA 1988;259:1185-9.

8 Leoung GS, Mills J, Hopwell PC, et al. Dapsonetrimethoprim for Pneumocystis carinii pneumonia in the acquired immunodeficiency syndrome. An Intern Med 1986;105:45-8.

9 Fischl MA, Richman DD, Grieco MH, et al. The efficacy of azidothymidine AZT) in the treatment of patients with AIDS and AIDS-related complex. $N$ Engl f Med 1987;317:185-91.

10 Creagh-Kirk T, Doi P, Andrews E et al. Survival experience among patients with AIDS receiving zidovudine. FAMA 1988;260:3009-15

11 Stambuk D, Youle M, Hawkins D, et al. The efficacy of azidothymidine (AZT) in the treatment of patients with AIDS and AIDS-related complex (ARC): an open uncontrolled treatment study. Qf Med 1989;262:161-74.

12 (ARC): an open uncontrolled treatment study. $Q$ M Med $1989,262: 161-74$. for acquired immuno-deficiency syndrome. MMWR 1987;36(suppl IS): I-15S

13 Armitage P, Berry G. Statistical methods in medical research Oxford: Blackwell Scientific Publications, 1987:429-33.

14 MacFadden DK, Hyland RH, Inouye T, Edelson JL, Rodriguez CH, Rebuck AS. Corticosteroids as adjunctive therapy in treatment of Pneumocystis carinii pneumonia in patients with acquired immunodeficiency syndrome. Lancet 1987; i: 1477-9. 
15 Peters BS, Bennett JM, Jeffries DJ, Knox K, Kocsis A, Pinching AJ Ineffectiveness of AL721 in HIV disease. Lancet 1990;335:545-6.

16 Rothenberg R, Woelfel $M$, Stoneburner R, Milberg J, Parker R, Truman B. Survival with the acquired immunodeficiency syndrome. $N$ Engl $f$ Med 1987;317:1297-302.

17 Gardner TD, Hanson PVJ, Griffiths PD, Collins JV, Gazzard BG. Diagnosis, treatment and outcome of pneumonia in the acquired immune deficiency syndrome. $\mathcal{F}$ Infect 1989;18:111-7.

18 Brenner M, Ognibene FP, Lack EE, et al. Prognostic factors and life expectancy of patients with acquired immunodeficiency syndrome and
Pneumocystis carinii pneumonia. Am Rev Respir Dis 1987;136:1199-206. 19 Penn I. The occurrence of malignant tumours in immunosuppressed states. Prog Allergy 1986;37:259-300

20 Peters BS, Matthews J, Gompels M, Hartley JC, Pinching AJ. Acut myeloblastic leukaemia in AIDS. AIDS 1990;4:367-8.

21 Swart AM, Weller I, Darbyshire JH. Early HIV infection: to treat or not to treat? BMF 1990;301:825-6.

\title{
Use of non-orthodox and conventional health care in Great Britain
}

\author{
Kate J Thomas, Jane Carr, Linda Westlake, Brian T Williams
}

\begin{abstract}
Objective-To describe the characteristics of patients using non-orthodox health care and their pattern of use of conventional health care with respect to a particular problem.
\end{abstract}

Design-Postal survey of all 2152 practitioners of acupuncture, chiropractic, homoeopathy, naturopathy, and osteopathy identified from 11 national professional association registers. Patients attending a representative sample of 101 responding practitioners completed questionnaires covering demographic characteristics, presenting problems, and use of the health service.

Setting-Practices of practitioners of nonorthodox health care in England, Scotland, and Wales.

Subjects-Qualified, non-medical practitioners of non-orthodox health care working in Great Britain and 2473 patients who had attended one of the sampled practitioners in an allocated time period between August 1987 and July 1988.

Results-An estimated 1909 practitioners were actively practising one of the study treatments in Great Britain in 1987. Of the estimated 70600 patients seen by this group of practitioners in an average week, most $(78 \%)$ were attending with a musculoskeletal problem. Two thirds of the patients were women. Only $2 \%$ were aged under 16 , but $15 \%$ were aged 65 or over. One in three patients had not received previous conventional care for their main problem; $18 \%$ were receiving concurrent nonorthodox and conventional care. Twenty two per cent of the patients reported having seen their general practitioner for any reason in the two weeks before the surveyed consultation.

Conclusions-Patients of non-orthodox health care, as provided by this group of practitioners, had not turned their backs on conventional health care. Non-orthodox treatment was sought for a limited range of problems and used most frequently as a supplement to orthodox medicine.

Medical Care Research

Unit, Department of Public Health Medicine,

University of Sheffield

Medical School, Sheffield

S10 2RX

Kate J Thomas, MA, senior

research associate

Jane Carr, BSC, research

assistant

Linda Westlake, BSC, statistician

Brian T Williams, FRCP, director

Correspondence to: Ms Thomas.

BMF 1991;302:207-10 ture, chiropractic, homoeopathy, and osteopathy considerable. Such training is being pursued both by medically qualified practitioners and by those without previous medical training.

In the United Kingdom, unlike in most other
European countries, a practitioner without a registered medical qualification may legally offer health care for payment and, at present, there is no formal regulation of practice. This state of affairs may change because of pressures from home and abroad for greater regulation and control. General Medical Council guidelines make possible referrals to practitioners who do not possess medical qualifications, provided that the referring medical practitioners retain responsibility for their patients. ${ }^{5}$ The extent of this responsibility requires clarification and is perceived as a problem by some general practitioners. ${ }^{6}$ At the same time some qualified, non-medical practitioners of the more established treatments are actively seeking statutory registration for their disciplines.

No statutory statistical information exists to inform the debates surrounding these issues. Our study goes some way to providing such data by describing the characteristics and behaviour of a national sample of non-orthodox health care practitioners and their patients and the way in which such patients make use of the two systems of health care over time and with respect to particular problems.

\section{Methods}

We focused on a particular type of non-orthodox care - that provided by qualified, non-medical practitioners belonging to national professional associations that regulate the practice of their members. All the practitioners in the study were trained in acupuncture, chiropractic, homoeopathy, medical herbalism, naturopathy, or osteopathy. The study entailed an initial postal survey, conducted in March 1987, of 2152 practitioners identified from the registers of 11 national professional associations representing practitioners in the six disciplines.

The second stage entailed a survey of the patients of a stratified random sample of 146 active practitioners, identified from the postal survey. The practitioners were stratified before sampling according to professional association membership and the length of time each practitioner had been in practice. A sample size of $5 \%$ was chosen for the six associations with membership over $100,10 \%$ for the four with membership under 100 , and $20 \%$ for the smallest association. All patients attending each of these practitioners were sampled during a specified time period allocated to each practitioner between August 1987 and July 1988. The duration of the sample period ranged from three days to two weeks, depending on the level of activity of each practitioner; a minimum of 25 patients per practitioner was sampled. The sample sizes were selected to ensure that sufficient numbers of patients were sampled within each of the diagnostic categories, thus producing a representative range of the problems presented to these non-orthodox health care practitioners. For each patient attending in the sample 